\title{
Solving for Estimated Temperature Inside Buildings by Finite Differential Method
}

\author{
Sirikul Siriteerakul and Teera Siriteerakul
}

\begin{abstract}
Temperature inside buildings depend on several factors such as type of wall material, shape and type of roof, position of mirror, etc. Structure or shape of building is also an important factor.

In this paper, we considered average temperature within three different structures of the buildings. We are considering buildings with the same height, base, perimeter and interior area of the building. However, the shape on the top are different.

To determine the lowest interior temperature, steady stage 2-D heat conduction equation in rectangular coordinate system was used in the assumption of homogeneous material, no convection nor radiation, and the buildings are in the same area and environment.

The lowest interior temperature were interested. It was found that building type 3 is the highest temperature of the interior area of the building, following by building 1 and building 2 , respectively. The temperature distribution in the building with more area on the top allow heat to pass more than the ones with less area.
\end{abstract}

Keywords - Finite Differential Method, Laplace's equation, temperature distribution.

\section{INTRODUCTION}

The differential equation is one of the most important tool that we can apply to solve many different fields of problems such as engineering and science, etc. Using the Finite differential method is easy and simple. It saves time to solve and helps us to solve the problem that can find the exact solution. Nantapol et.al [1] have used Finite differential method to find an estimated solution and an error of parabolic equation which is one of the differential equations. Sirikul et.al simulated heat transfer in the model houses with the difference roof geometry [3], type of wall material [4], shape of the building [5] and glass wall arrangement patterns [6] .etc.

The objective of this paper is to study the temperature inside the three different shape buildings with different area on the top by using finite difference method, central difference, and the buildings are made from the same material and have the same area, height and the external temperature.

Sirikul Siriteerakul, Department of Mathematics, Faculty of Science, King Mongkut's Institute of Technology Ladkrabang, Chalongkrung Street, Bangkok, Thailand..

Teera Siriteerakul, Department of Mathematics, Faculty of Science, King Mongkut's Institute of Technology Ladkrabang, Chalongkrung Street, Bangkok, Thailand.

\section{GOVERNING EQUATION AND METHODOLOGY}

The heat conduction in two dimensional rectangular coordinate system at steady stage or Laplace's equation was computed under the assumption of close area without convection or radiation. It can be expressed as:

$$
\frac{\partial^{2} T}{\partial x^{2}}+\frac{\partial^{2} T}{\partial y^{2}}=0
$$

\section{A. Finite difference method}

Finite difference method is the simplest numerical method for approximating the differential equation.

For equally divided grid, there are 3 types of Finite difference method. [2]

\section{Forward difference}

$$
\left.\frac{\partial^{2} u}{\partial x^{2}}\right|_{x_{i}}=\frac{u\left(x_{i+2}, y_{i}\right)-2 u\left(x_{i+1}, y_{i}\right)+u\left(x_{i}, y_{i}\right)}{h^{2}}
$$

2. Backward difference

$$
\left.\frac{\partial^{2} u}{\partial x^{2}}\right|_{x_{i}}=\frac{u\left(x_{i}, y_{i}\right)-2 u\left(x_{i-1}, y_{i}\right)+u\left(x_{i-2}, y_{i}\right)}{h^{2}}
$$

\section{Central difference}

$$
\left.\frac{\partial^{2} u}{\partial x^{2}}\right|_{x_{i}}=\frac{u\left(x_{i+1}, y_{i}\right)-2 u\left(x_{i}, y_{i}\right)+u\left(x_{i-1}, y_{i}\right)}{h^{2}}
$$

These are explicit schemes. In this problem, the central difference provide an appropriate approximation rather than forward difference and backward difference in the case of equally divided grid.

Area on plane $x y$ were divided to small-square by $\Delta x$ and $\Delta y$ are constant. Shown as grid space system in figure below.

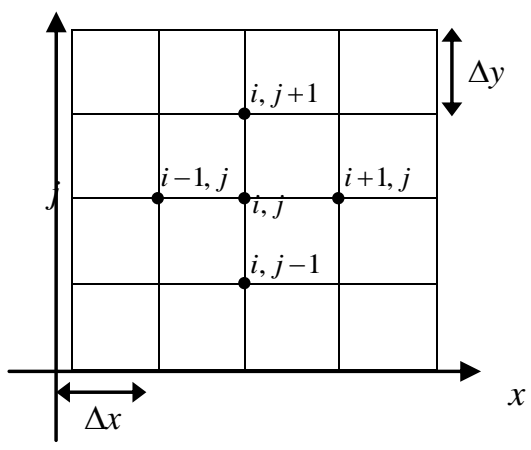


The governing equation was estimated the derivatives by using Talor series expansion as follows:

$$
\begin{aligned}
& \frac{\partial^{2} T}{\partial x^{2}}=\frac{1}{(\Delta x)^{2}}\left[T_{i-1, j}-2 T_{i, j}+T_{i+1, j}\right] \\
& \frac{\partial^{2} T}{\partial y^{2}}=\frac{1}{(\Delta y)^{2}}\left[T_{i, j-1}-2 T_{i, j}+T_{i, j+1}\right]
\end{aligned}
$$

After substitution of equation (5) and (6) in Laplace's equation, eq. (1), we obtained

$\frac{1}{(\Delta x)^{2}}\left[T_{i-1, j}-2 T_{i, j}+T_{i+1, j}\right]+\frac{1}{(\Delta y)^{2}}\left[T_{i, j-1}-2 T_{i, j}+T_{i, j+1}\right]=0$

$\left(\frac{\Delta y}{\Delta x}\right)^{2}\left[T_{i-1, j}-2 T_{i, j}+T_{i+1, j}\right]+\left[T_{i, j-1}-2 T_{i, j}+T_{i, j+1}\right]=0$

For $\alpha=\left(\frac{\Delta y}{\Delta x}\right)^{2}$

$\alpha T_{i-1, j}-2 \alpha T_{i, j}+\alpha T_{i+1, j}+T_{i, j-1}-2 T_{i, j}+T_{i, j+1}=0$

$\left[\alpha T_{i-1, j}-2(\alpha+1) T_{i, j}+\alpha T_{i+1, j}\right]+T_{i, j-1}+T_{i, j+1}=0$

Pattern form of Laplace's equation when using the second order central difference will be represent as follows

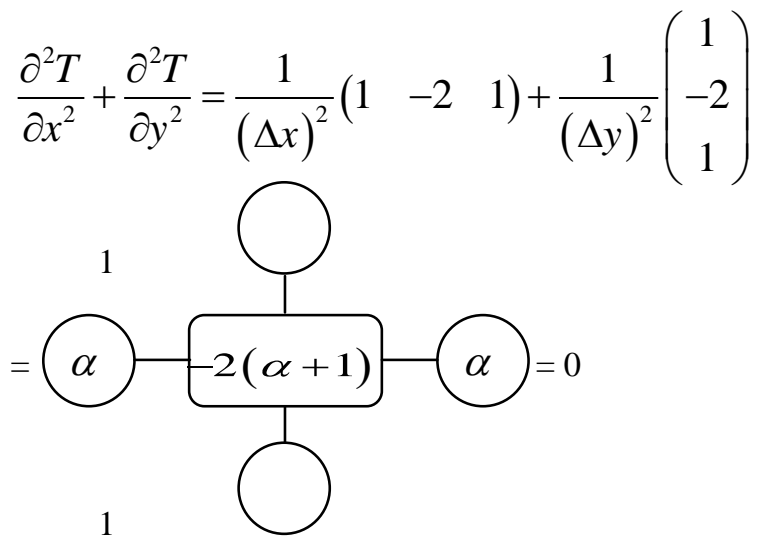

If $\Delta x=\Delta y$ then $\alpha=1$ therefore

$$
T_{i-1, j}-4 T_{i, j}+T_{i+1, j}+T_{i, j-1}+T_{i, j+1}=0
$$

Write it in the matrix form, then we solved the linear equation system by Mathematica.

\section{PROBLEM SPECIFICATION}

\section{A. Procedures}

Step 1 Plot a building shape on a graph, then divide into small shape of rectangles.

Step 2 Determines the ordered pair $(i, j)$ for each node.

Step 3 Consider every interior node. Start with node $(1,1)$, Substitute known value into the pattern equation. We will have a system of linear equations.

Step 4 Solve a linear equation system by using the matrix form. In this paper, we use Mathematica to solve the system.

Step 5 Find the average temperature of the symmetry axis of the building and compare to the other differential shape of the building.

\section{B. Problems and Solutions}

Problem 1: Assume that external temperature is 25 Degree Celsius at the ground and 33 Degree Celsius in the air which shown in the Figure 1. Find 71 node of unknown interior temperature

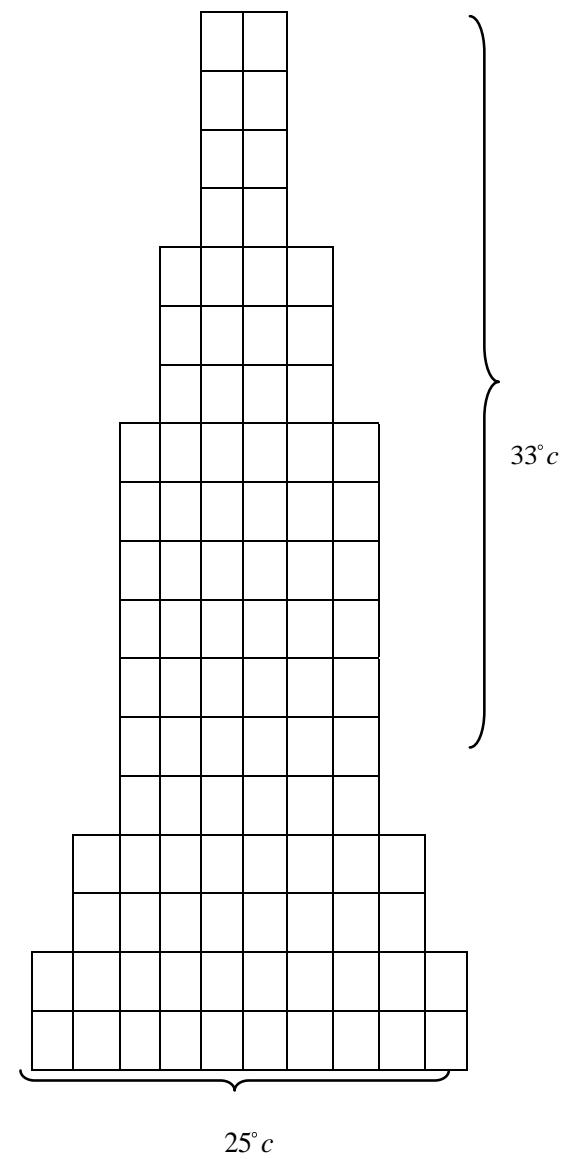

Fig. 1: Structure of building type 1

Find the temperature at $(1,1)$

From equation (1), substitute known value.

$T_{0,1}=33^{\circ} \mathrm{c}, T_{1,0}=25^{\circ} \mathrm{c}, T_{1,2}=33^{\circ} \mathrm{c}$

we obtained

$$
\begin{gathered}
T_{0,1}-4 T_{1,1}+T_{2,1}+T_{1,0}+T_{1,2}=0 \\
33-4 T_{1,1}+T_{2,1}+25+33=0 \\
-4 T_{1,1}+T_{2,1}=-91
\end{gathered}
$$

Find the temperature at $(2,1)$

From equation (1), substitute known value.

$$
\begin{aligned}
& T_{1,1}-4 T_{2,1}+T_{3,1}+T_{2,0}+T_{2,2}=0 \\
& T_{1,1}-4 T_{2,1}+T_{3,1}+25+T_{2,2}=0
\end{aligned}
$$




$$
T_{1,1}-4 T_{2,1}+T_{2,2}+T_{3,1}=-25
$$

Find the temperature at $(2,2)$

From equation (1), $T_{1,2}=33^{\circ} \mathrm{C}$

substitute known value. we will get

$$
\begin{gathered}
T_{1,2}-4 T_{2,2}+T_{3,2}+T_{2,1}+T_{2,3}=0 \\
33-4 T_{2,2}+T_{3,2}+T_{2,1}+T_{2,3}=0 \\
T_{2,1}-4 T_{2,2}+T_{2,3}+T_{3,2}=-33
\end{gathered}
$$

Do the same procedure until the last unknown node. After that write those equations in the matrix form $A x=B$

$$
\left[\begin{array}{ccccc}
4 & -1 & 0 & \cdots & 0 \\
-1 & 4 & -1 & \cdots & 0 \\
0 & -1 & 4 & \cdots & 0 \\
\vdots & \vdots & \vdots & \ddots & \vdots \\
0 & 0 & 0 & \cdots & 4
\end{array}\right]\left[\begin{array}{c}
T_{1,1} \\
T_{2,1} \\
T_{2,2} \\
\vdots \\
T_{9,1}
\end{array}\right]=\left[\begin{array}{c}
91 \\
25 \\
33 \\
\vdots \\
91
\end{array}\right]
$$

Problem 2: Assume that external temperature is 25 Degree Celsius at the ground and 33 Degree Celsius in the air which shown in the Figure 2. Find 71 node of unknown interior temperature

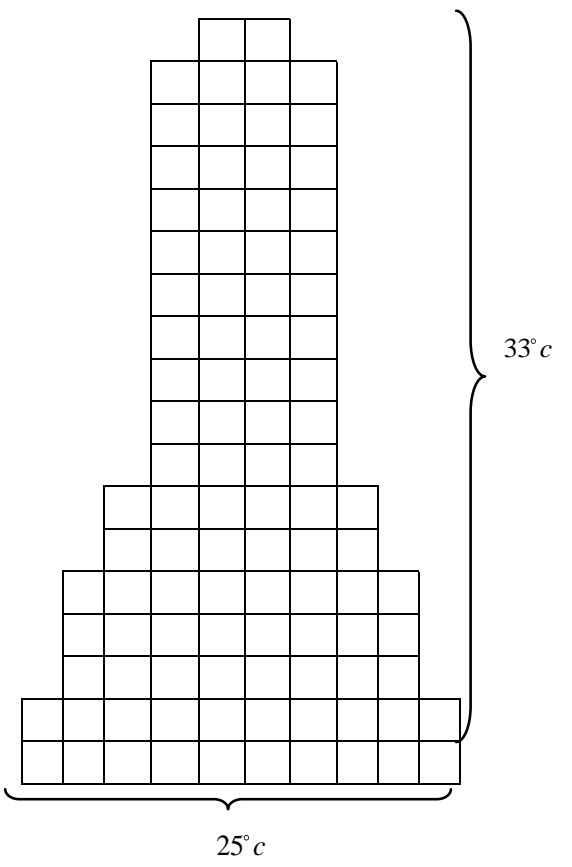

Fig. 2: Structure of building type 2

Find the temperature at $(1,1)$

From equation (1), substitute known value. $T_{0,1}=33^{\circ} \mathrm{c}$,

$$
\begin{aligned}
& T_{1,0}=25^{\circ} c, T_{1,2}=33^{\circ} c \text { we obtained } \\
& T_{0,1}-4 T_{1,1}+T_{2,1}+T_{1,0}+T_{1,2}=0 \\
& 33-4 T_{1,1}+T_{2,1}+25+33=0 \\
& -4 T_{1,1}+T_{2,1}=-91
\end{aligned}
$$

Find the temperature at $(2,1)$

From equation (1), substitute known value.

$$
\begin{aligned}
& T_{1,1}-4 T_{2,1}+T_{3,1}+T_{2,0}+T_{2,2}=0 \\
& T_{1,1}-4 T_{2,1}+T_{3,1}+25+T_{2,2}=0 \\
& T_{1,1}-4 T_{2,1}+T_{2,2}+T_{3,1}=-25
\end{aligned}
$$

Find the temperature at $(2,2)$

From equation (1), substitute known value.

$$
\begin{aligned}
& T_{1,2}-4 T_{2,2}+T_{3,2}+T_{2,1}+T_{2,3}=0 \\
& 33-4 T_{2,2}+T_{3,2}+T_{2,1}+T_{2,3}=0 \\
& T_{2,1}-4 T_{2,2}+T_{2,3}+T_{3,2}=-33
\end{aligned}
$$

Do the same procedure until the last unknown node. After that write those equations in the matrix form $A x=B$

Problem 3: Assume that external temperature is 25 Degree Celsius at the ground and 33 Degree Celsius in the air which shown in the Figure 3. Find 71 node of unknown interior temperature.

Find the temperature at $(1,1)$

From equation (1), substitute known value.

$$
\begin{gathered}
T_{0,1}=33^{\circ} c, T_{1,0}=25^{\circ} c, T_{1,2}=25^{\circ} c \text { we obtained } \\
T_{0,1}-4 T_{1,1}+T_{2,1}+T_{1,0}+T_{1,2}=0 \\
33-4 T_{1,1}+T_{2,1}+25+25=0 \\
-4 T_{1,1}+T_{2,1}=-83
\end{gathered}
$$

Find the temperature at $(2,1)$

From equation (1), substitute known value.

$$
\begin{aligned}
& T_{2,0}=25^{\circ} c \text { we will get } \\
& T_{1,1}-4 T_{2,1}+T_{3,1}+T_{2,0}+T_{2,2}=0 \\
& T_{1,1}-4 T_{2,1}+T_{3,1}+25+T_{2,2}=0 \\
& T_{1,1}-4 T_{2,1}+T_{2,2}+T_{3,1}=-25
\end{aligned}
$$

Do the same procedure until the last unknown node. After that write those equations in the matrix form $A x=B$ 


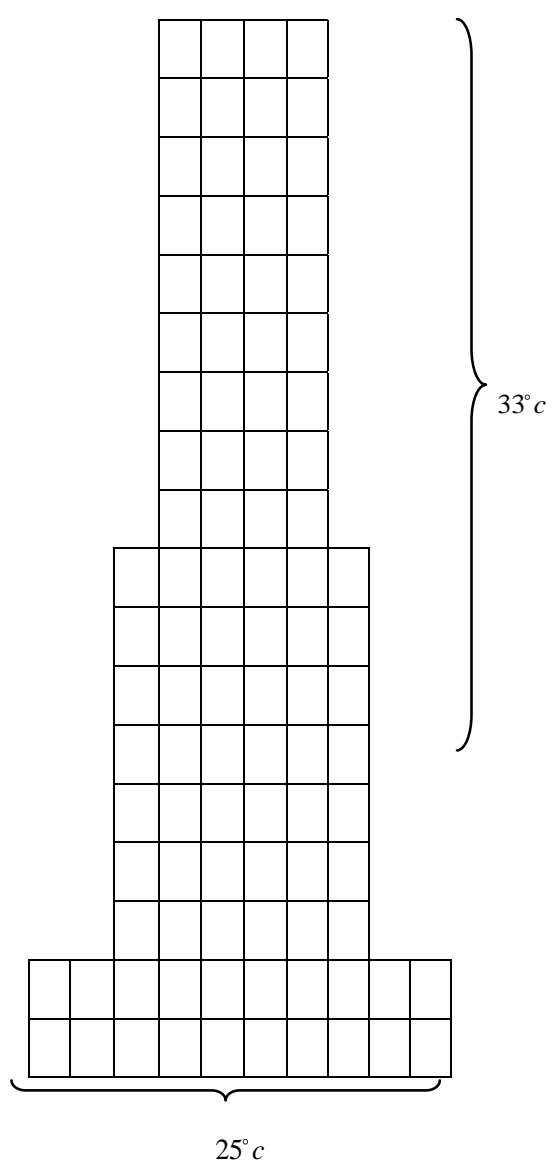

Fig. 3: Structure of building type 3

\section{REsUlt}

Solve the linear systems by using "Mathematica". The result of each problem are shown as follow:

Problem 1:

$$
\left[\begin{array}{c}
T_{1,1} \\
T_{2,1} \\
T_{2,2} \\
\vdots \\
T_{9,1}
\end{array}\right]=\left[\begin{array}{c}
29.8060 \\
28.2238 \\
30.6455 \\
\vdots \\
29.8060
\end{array}\right]
$$

Problem 2:

$$
\left[\begin{array}{c}
T_{1,1} \\
T_{2,1} \\
T_{2,2} \\
\vdots \\
T_{9,1}
\end{array}\right]=\left[\begin{array}{c}
29.7962 \\
28.1847 \\
30.5480 \\
\vdots \\
29.7962
\end{array}\right]
$$

Problem 3:

$$
\left[\begin{array}{c}
T_{1,1} \\
T_{2,1} \\
T_{3,1} \\
\vdots \\
T_{9,1}
\end{array}\right]=\left[\begin{array}{c}
30.0004 \\
29.0015 \\
28.0057 \\
\vdots \\
30.0004
\end{array}\right]
$$

After that the average temperature along the symmetry axis of the buildings are computed and compared to the other differential shape of the building.

We found that the average temperature at the symmetry axis building type 1 is equal to 31.9584 Degree Celsius, building type 2 is equal to 31.9500 Degree Celsius and building type 3 is equal to 32.1066 Degree Celsius. So building type 3 has the highest average temperature then building type 1 and building type 2 is the lowest which shown in Figure 4.

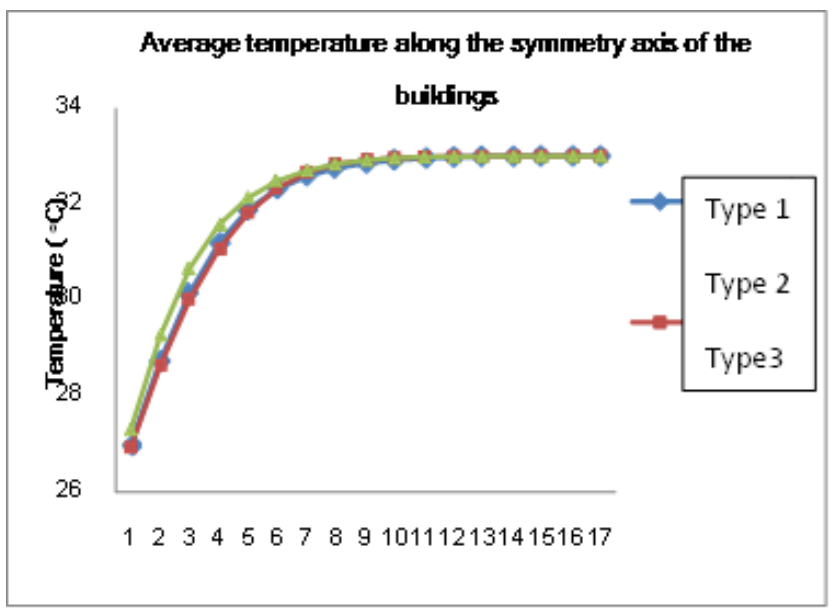

Fig. 4: Temperature at the symmetry axis building 1, building 2 and building 3

\section{CONCLUSION}

Computing of temperature at each point of the buildings and average of temperature at symmetry axis were done. The results showed difference, especially type 3 which has most area on the top. It was found that building type 3 is the highest temperature, following by building 1 and building 3 , respectively. The temperature distribution in the building with more area on the top can more heat pass than less area.

\section{REFERENCES}

[1] Nantapol et.al Ramkamhang Journal (Science and Technology), pp. 55-64.

(http://www.rd.ru.ac.th/images/Journal/Sci/15_2/SCI\%2055\%2002\%20 05.pdf) https://doi.org/10.1002/pts.568

[2] Pakkinee Chitsakul. Partial Differential Equation. Bangkok : Department of Mathematics, Faculty of Science, King Mongkut's Institute of Technology Ladkrabang.

[3] Sirikul Siriteerakul, Wiphada Chumnan and Teera Siriteerakul, "Temperature Distribution in three Model Houses with Different Roof Geometries", Journal of the Automation and Control Engineering (JOACE), Vol.2, No.1,(2014), 71-74. 
15th PATTAYA-THAILAND Int'I Conference on Advances in Science, Engineering \& Technology (PASET-19) Aug. 12-14 2019 Pattaya (Thailand)

https://doi.org/10.12720/joace.2.1.71-74

[4] Sirikul Siriteerakul and Teera Siriteerakul, "Heat Conduction Analysis of Wall Materials", Proceeding of the International Conference on Engineering Science and Applications (ICESA), Vol.1, 2017, 63-74.

[5] P. Chitsakul and S. Sirikul, Simulation of Heat Transfer within Geometry Shape Symmetry Building,TJIA 2009.
[6] S. Sirikul and S. Thenissara, Glass Arrangement Effect on Heat Transfer in Rectangular Building, WASET, 2013. 